\title{
Luminosity Function of Some Open Clusters
}

\author{
W. H. Elsanhoury, ${ }^{1}$ M. A. Hamdy, ${ }^{1}$ M. I. Nouh, ${ }^{2}$ A. S. Saad, ${ }^{3}$ and S. M. Saad ${ }^{1}$ \\ ${ }^{1}$ Departement of Astronomy, National Research Institute of Astronomy and Geophysics (NRIAG), Cairo 11421, Egypt \\ ${ }^{2}$ Department of Physics, Faculty of Science, Northern Border University, Arar 1321, Saudi Arabia \\ ${ }^{3}$ Department of Mathematics, Qassim University, Qassim 51452, Saudi Arabia
}

Correspondence should be addressed to M. I. Nouh, abdo_nouh@hotmail.com

Received 11 January 2011; Accepted 21 February 2011

Academic Editors: G. Chernov and M. Marconi

Copyright ( $) 2011$ W. H. Elsanhoury et al. This is an open access article distributed under the Creative Commons Attribution License, which permits unrestricted use, distribution, and reproduction in any medium, provided the original work is properly cited.

We investigated the luminosity function (LF) and initial mass function (IMF) of some open clusters having different ages. To calculate the LF, we followed the classical definition by van Rhijn (1936). Statistical investigation of the dispersion around a range of magnitudes concerning what is called Wielen dip revealed that the dip is unreal. To confirm the unreality of the dip, we computed the IMF for these open clusters, the statistical investigation of the IMF confirmed the results obtained using the LF, that is, there is no dip for these open clusters under study.

\section{Introduction}

Open clusters constitute an important part of a process transforming gas and dust into stars. They are observed as the most prominent parts in the regions of active star formation, or as tracers of the ceased star formation process in the general Galactic field. However, the role they are playing in this process has still not been fully understood. In spite of their prominence, there are indications that classical open clusters contribute only $10 \%$ or even less input [1-3] to the total stellar population of the Galactic disc [4].

The LF is among the most important tools to analyze the composition and evolution of stellar systems and is especially effective in studies of open star clusters, since the absolute magnitudes and ages of their constituent stars can be determined fairy accurately.

Wielen [5] derived the LF $(\phi)$ for solar neighborhood stars with aid of Gliese's catalogue $[6,7]$ by counting Gliese's stars in appropriate volumes of space. In this work, the unit of $\phi$ is stars per unit magnitude interval in a complete sphere of radius $r=20 \mathrm{pc}$.

Miller and Scalo [8] presented a comparison between McCuskey [9], Luyten [10], and Wielen [5] LFs along the LF adopted by him. The LF of Wielen and McCuskey shown a small dip around $M_{V}=7$. Miller and Scalo [8] attach there is no physical significance of this dip because (a) its size is within the errors of the determination of the LF and (b) this dip is not found by all investigators.

Lee and Chun [11] selected some of open the clusters (22 open clusters) and 3 associations on the following criteria: (i) correct photometric observations, (ii) easy selection of member stars, (iii) wide range of magnitude and mass, and (iv) large number of MS stars, taking into account the brightening effect of bright MS stars. Using these objects, they derived the LF and mass function MF. The results obtained were consistent with solar neighborhood stars by Wielen [5].

Lee and Chun [11] indicated the presence of a dip at $M_{V} \sim 7$ mag. or $\log \mathcal{M} \sim-0.2$ in open clusters, which were studied in his paper, similar to what was called Wielen dip which appears in solar neighborhood stars in spite of the fact that Wielen, in his study [5] using solar neighborhood stars did not mention the existence of any dip. Moreover, Lee and Chun [11] mentioned that conclusive confirmation of the existence of this dip in the cluster initial mass function IMF must be made by using many LFs of open clusters older than Hyades because the possibility of the turnover of cluster LF at low mass $\left(\mathcal{M}<1 \mathcal{M}_{\odot}\right)$ has been suggested $[12,13]$.

Lee and Sung [14] studied the LF for the Pleiades cluster, showing what they called Wielen dip at $M_{V}=7 \mathrm{mag}$. Also, 
TABle 1: Basic data.

\begin{tabular}{lccccc}
\hline Cluster name & RA 2000 h. m. s. & Dec 2000 d. m. s. & Range of magnitudes & Range of distances (pc) & $N$ (Known member stars) \\
\hline Stock 2 & 0214.4 & +5916 & $-3.115-12.709$ & $4.371-5000$ & 85 \\
NGC 869 & 0219.1 & +5709 & $-7.114-14.502$ & $3.0185-10000$ & 152 \\
NGC 884 & 0222.0 & +5708 & $-4.338-14.502$ & $3.0185-2631.579$ & 146 \\
NGC 2168 & 0609.1 & +2421 & $2.3018-11.883$ & $5.30814-181.8182$ & 34 \\
NGC 2264 & 0641.0 & +0953 & $-3.383-11.017$ & $12.361-4347.8261$ & 28 \\
NGC 2516 & 0758.1 & -6045 & $-2.611-11.191$ & $9.1491-3333.333$ & 45 \\
NGC 2632 & 0840.4 & +1941 & $-0.815-12.22$ & $5.96338-1492.5373$ & 63 \\
NGC 3532 & 1106.0 & -5844 & $-2.436-11.68$ & $6.32511-709.21986$ & 50 \\
Ruprecht 147 & 1916.7 & -1618 & $0.16625-10.8993$ & $7.2674-413.223$ & 26 \\
\hline
\end{tabular}

TABLE 2: Stock 2 double frequency table.

\begin{tabular}{|c|c|c|c|c|c|c|c|c|c|}
\hline Mag (absolute mag.). & 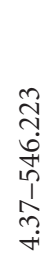 & 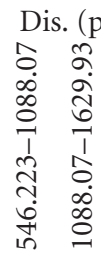 & 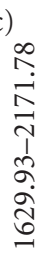 & 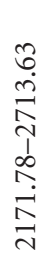 & 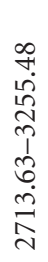 & 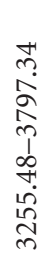 & $\begin{array}{l}\stackrel{a}{a} \\
\stackrel{m}{m} \\
\stackrel{+}{+} \\
\stackrel{n}{n} \\
\text { n}\end{array}$ & 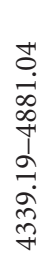 & 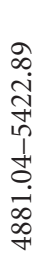 \\
\hline$-3.115-1.399$ & & & & & & & 1 & & 2 \\
\hline$-1.399-0.317$ & 3 & 1 & & & & & & & \\
\hline $0.317-2.034$ & 7 & 1 & & & & & & & \\
\hline $2.034-3.750$ & 6 & & & & & & & & \\
\hline $3.750-5.467$ & 13 & & & & & & & & \\
\hline $5.467-7.183$ & 13 & & & & & & & & \\
\hline $7.183-8.899$ & 22 & & & & & & & & \\
\hline $8.99-10.616$ & 9 & & & & & & & & \\
\hline $10.616-12.332$ & 6 & & & & & & & & \\
\hline $12.335-14.049$ & 1 & & & & & & & & \\
\hline
\end{tabular}

TABLE 3: Stock 2 luminosity function calculations.

\begin{tabular}{lcc}
\hline Mean magnitude & $\begin{array}{c}\text { Density }(\mathrm{LF}) \\
\phi\left(M_{V}\right)\end{array}$ & $\log \phi\left(M_{V}\right)$ \\
\hline-2.257 & $1.331 \times 10^{-11}$ & -10.876 \\
-0.5417 & $2.684 \times 10^{-9}$ & -8.571 \\
1.176 & $6.0976 \times 10^{-9}$ & -8.215 \\
2.892 & $5.121 \times 10^{-9}$ & -8.291 \\
4.608 & $1.1095 \times 10^{-8}$ & -7.955 \\
6.325 & $1.1095 \times 10^{-8}$ & -7.955 \\
8.041 & $1.876 \times 10^{-8}$ & -7.727 \\
9.758 & $7.681 \times 10^{-9}$ & -8.1146 \\
11.474 & $5.121 \times 10^{-9}$ & -8.2906 \\
13.191 & $8.534 \times 10^{-10}$ & -9.069 \\
\hline
\end{tabular}

Lee et al. [15] studied the LF for the Hyades and Praesepe clusters. According to this study, a Wielen dip was clearly seen at $M_{V}=9$ mag. (more precisely at $M_{V}=8.5 \sim 9$ mag.). This dip is about 2 mag. fainter than the case of the Pleiades cluster whose Wielen dip position is consistent with that for the solar neighborhood field stars.
TABLE 4: Stock 2 LF.

\begin{tabular}{lccccc}
\hline$M_{V}$ & -2.257 & -0.5417 & 1.176 & 2.892 & 4.608 \\
$\log \phi\left(M_{V}\right)$ & -10.876 & -8.571 & -8.215 & -8.291 & -7.955 \\
\hline$M_{V}$ & 6.325 & 8.041 & 9.758 & 11.474 & 13.191 \\
$\log \phi\left(M_{V}\right)$ & -7.955 & -7.727 & -8.1146 & -8.2906 & -9.069 \\
\hline
\end{tabular}

Belikov et al. [16] studied the fine structure of Pleiades LF and premain sequence evolution, they found that there are three features (dips) in the observed cluster LF in a magnitude range $M_{V}=5 \mathrm{mag} .-12 \mathrm{mag}$. The first dip together with the adjacent maximum has a pre-MS nature. In their study of the theoretical LFs construction and comparing with the observations, they argued that since the LF depends on both IMF and mass-luminosity relation MLR derivative and since MLR of pre-MS stars differs from that of MS stars, the pre-MS branch (in the cluster where it is present) should affect the LF. Especially, a sharp contrast is expected at (or just below) the turn on the point at the transition between MS and pre-MS branches. According to Piskunov et al. [17], this transition produces a local "bump" in the LF followed by a dip (herein they call this "bump" as "H-peak" and the effect of peak and dip existence as "Hfeature": they chose this abbreviation to stress that the feature is related to the beginning of hydrogen burning as a star approaches the ZAMS) while the two dips (at $M_{V}=7.5 \mathrm{mag}$. and $M_{V}=9.5$ mag.) are assumed to be field LF features: Wielen and Kroupa et al. (WK for short) dips, into which theoretical models fail to reproduce them.

In the study of the low-luminosity stellar MF, Kroupa et al. [18] discussed possible mechanisms causing the WK dips. They proposed that both details of the LF are the result of mass-luminosity relation fine structure generated by the equation of state and the opacity law. They proposed this idea by causing low-mass ZAMS models and showed that the Kroupa et al. dip is caused by the influence of $\mathrm{H}_{2}$ molecules on the equation of state. They attributed the Wielen dip to the increasing importance of $\mathrm{H}^{-}$ions as an opacity source. Belikov et al. in [16] using the more sophisticated models of D'Antona and Mazzitelli [19], concluded that these dips did not appear in their results.

In the present paper, we tried to study the LF of the some open clusters of different ages and magnitudes, with 
TABLE 5: NGC 869 (h-Per) LF.

\begin{tabular}{lccccccc}
\hline$M_{V}$ & -6.237 & -4.484 & -2.731 & -0.977 & -0.776 & 2.52915 & 4.282 \\
$\log \phi\left(M_{V}\right)$ & -12.264 & -9.317 & -9.106 & -8.858 & -8.687 & -8.643 & -8.451 \\
\hline$M_{V}$ & 6.036 & 7.790 & 9.542 & 11.296 & 13.049 & 14.802 \\
$\log \phi\left(M_{V}\right)$ & -8.297 & -8.342 & -8.150 & -8.255 & -9.121 & -9.597 & \\
\hline
\end{tabular}

TABLe 6: NGC $884(\chi-P e r)$ LF.

\begin{tabular}{lccccccc}
\hline$M_{V}$ & -3.558 & -2 & -0.44 & -1.12 & 2.678 & 4.238 & 5.797 \\
$\log \phi\left(M_{V}\right)$ & -8.759 & -8.629 & -8.165 & -7.921 & -7.301 & -6.756 & -6.641 \\
\hline$M_{V}$ & 7.356 & 8.915 & 10.474 & 12.034 & 13.593 & 15.152 \\
$\log \phi\left(M_{V}\right)$ & -6.59 & -6.465 & -6.448 & -6.804 & -7.846 & -7.846 \\
\hline
\end{tabular}

TABLe 7: NGC 2168 (M35) LF.

\begin{tabular}{|c|c|c|c|c|c|c|c|}
\hline$M_{V}$ & 3.081 & 4.641 & 6.2 & 7.759 & 9.318 & 10.877 & 12.437 \\
\hline $\log \phi\left(M_{V}\right)$ & -7.244 & -6.081 & -5.553 & -4.362 & -4.768 & -5 & -5.467 \\
\hline
\end{tabular}

TABLE 8: NGC 2264 (S Mon) LF.

\begin{tabular}{lccccrr}
\hline$M_{V}$ & -2.023 & 0.699 & 3.42 & 6.141 & 8.863 & 11.584 \\
$\log \phi\left(M_{V}\right)$ & -9.8 & -9.118 & -9.039 & -9.118 & -9.04 & -9.817 \\
\hline
\end{tabular}

TABLE 9: NGC 2516 LF.

\begin{tabular}{lccccccc}
\hline$M_{V}$ & -1.583 & 0.475 & 2.532 & 4.590 & 6.647 & 8.705 & 10.763 \\
$\log \phi\left(M_{V}\right)$ & -8.335 & -8.541 & -8.345 & -8.047 & -8.269 & -8.093 & -8.445 \\
\hline
\end{tabular}

Table 10: NGC 2632 (Praesepe, M44) LF.

\begin{tabular}{lcccccccc}
\hline$M_{V}$ & 0.006 & 1.648 & 3.290 & 4.933 & 6.575 & 8.217 & 9.859 & 11.501 \\
$\log \phi\left(M_{V}\right)$ & -7.016 & -6.744 & -6.734 & -7 & -6.793 & -6.851 & -7.395 & -7.094 \\
\hline
\end{tabular}

TABLe 11: NGC 3532 LF.

\begin{tabular}{lcccccccc}
\hline$M_{V}$ & -1.438 & 0.559 & 2.555 & 4.551 & 6.548 & 8.544 & 10.540 & 12.537 \\
$\log \phi\left(M_{V}\right)$ & -8.046 & -7.602 & -7.59 & -6.314 & -5.995 & -6.092 & -6.217 & -6.995 \\
\hline
\end{tabular}

TABLE 12: Ruprecht 147 LF.

\begin{tabular}{lcccccc}
\hline$M_{V}$ & 1.199 & 3.265 & 5.330 & 7.396 & 9.461 & 11.527 \\
$\log \phi\left(M_{V}\right)$ & -7.729 & -7.034 & -6.254 & -5.828 & -6.032 & -6.129 \\
\hline
\end{tabular}

concentration on magnitude range $6 \leq M_{V} \leq 9$ (the range of the so called Wielen dip) in order to

(i) test the reality of the presence of this dip (i.e., Wielen dip) in open cluster,

(ii) to find the correct position of that dip, if present, and its dependence on cluster age.

\section{Observational Materials}

If the number of stars is increased in the study of luminosity function of open clusters, the statistical weight can be increased, reducing the possible selection effort. This general approach can be achieved only if the each cluster has statistically enough member stars. Accordingly, the criteria of the selection process of the open clusters to be included in this study to meet the goals that must be achieved were as follows: (a) the open cluster must include enough numbers of most probable members that cover the range of mag. $\left(6 \leq M_{V} \leq 9\right)$, and (b) the most probable member stars in the cluster are determined from proper motion and position.

In our study of the local population of Galactic open clusters, we obtained data that now allow a reliable construction of their luminosity and mass functions. Our cluster sample identified in the all-sky compiled catalogue ASCC-2.5 [20]. For each star projected on a cluster area, a membership 
TABLE 13: The dispersions of the IMF of the whole cluster compared with that of the dispersions around the dip region.

\begin{tabular}{lcc}
\hline Cluster name & $\begin{array}{c}\text { Dispersion of the } \\
\text { LF curve of the } \\
\text { whole cluster }\end{array}$ & $\begin{array}{c}\text { Dispersion around } \\
\text { the dip region }\end{array}$ \\
\hline Stock 2 & 0.912 & 0.21 \\
NGC 869 (h-Per) & 1.076 & 0.1 \\
NGC 884 ( -Per) & 0.837 & 0.095 \\
NGC 2168 (M35) & 0.954 & 0.605 \\
NGC 2264 S Mon & 0.379 & 0.046 \\
NGC 2516 & 0.18 & 0.176 \\
Praesepe NGC 2632 & 0.223 & 0.332 \\
(M44) & 0.807 & 0.111 \\
NGC 3532 & 0.730 & 0.212 \\
Ruprecht 147 & &
\end{tabular}

probability was determined in an iterative that takes spatial, photometric, and proper motion distributions of stars into account within the corresponding area on the sky [21]. At the end of the iterations for each cluster, we obtained new coordinates of the cluster center, the cluster size, the mean proper motion, the distance from the Sun, reddening, and age $[22,23]$. These parameters were determined with data on the most probable members, for example, stars having kinematic membership probability higher than 50\%. The results are included in the catalogue of open cluster data $(C O C D)$ and its extension $[22,23]$. This completeness limit corresponds to a range of magnitude $6 \leq M_{V} \leq 9$. Table 1 shows the basic data of 9 open clusters selected according to the above criteria.

\section{Method of Analysis}

The LF in our analysis was determined using the classical method of Van Rhijn [24]. It is defined as the number of stars in certain magnitude interval per unit volume. The volume and magnitude elements were determined by dividing the cluster into shells with certain width depending on the number of the stars in each cluster.

Double frequency tables of the number of stars in each radius interval (shell) corresponding to each magnitude interval were established. In order to determine the LF from the double frequency table, we counted the number of stars which fall in the magnitude interval corresponding to each distance interval, then we divided the counts in each shell by the volume of this shell (i.e. density) and constructed a table representing the mean absolute magnitude and density $\phi\left(M_{V}\right)$. Table 2 represents a sample of double frequency table for Stock 2 as an example, into which the number of members is 85 , distributed into equal magnitude intervals (i.e., absolute magnitude) and distance intervals (measuring in parsec unit).

It was found that 80 members are included in the distance interval $(4.370 ; 546.223 \mathrm{pc})$, the remaining 5 members were distributed in other intervals as shown in the table, according to the statistical distribution of the data. Using the double frequency table, we calculated the LF as it is represented in Table 3 according to Van Rhijn's [24] definition of LF.

\section{Results}

4.1. The Luminosity Function. Depending on the previously mentioned statistical method, the LF of the studied clusters of our interest were calculated. The LF of the clusters studied are tabulated in Tables 4, 5, 6, 7, 8, 9, 10, 11, and 12 .

NGC 2264, 2168, and Praesepe had been studied before by Lee et al. in 1984, the data used were selected on basis of photometric observations and proper motion studies. The LF and MF of these clusters are different with cluster age. On the other hand, other clusters (except Ruprecht 147) had been also studied by Lee and Chun in 1988. According to these studies, the combined LFs of open clusters and associations, the initial luminosity function ILF, and initial mass function IMF of these clusters were derived. They show slight differences in the bright and faint parts as compared with the field star ILF and IMF. However, the general shapes of the cluster ILF and IMF agree well with those for field stars in the solar neighborhood. We note that, all of these above studies $[11,25]$ depend on counting the number of stars within certain magnitude interval, regardless of the classical definition adopted by Van Rhijn [24].

Recently, Kalirai et al. [26] studied luminosity functions for NGC 2168: the LF rises until $M_{V}=5$ and then dips down at $M_{V}=7$ and rises again. This dip was most likely the result of poor statistics or cluster stars being subtracted off in the blank field and not physical. In that sense, Kalirai et al. [26]: stated that we do note that the luminosity function given in Barrado Y Navascués et al. [27] does not show a dip at this magnitude. This was constructed by using the $I$ band LF in Table 3 of Barrado et al. [27] and correcting to $V$ by using the empirical fiducial in Table 1 of that paper.

Sanner and Geffert [28] studied the fields of nine open clusters (e.g., Stock 2, Pleiades, and Praesepe), based on Tycho2 catalogue. They determined membership probabilities for the stars in the cluster fields from the stellar proper motions and used the Tycho-2 photometry to compute the initial mass function (IMF) for the clusters from the main sequence turnoff point down to approximately. $1 M_{\odot}$.

In this work, the computed LFs of the studied open clusters are represented in Figure 1. From the general appearance of the curves in this figure, it is so difficult to conclude whether there is a dip or not.

In order to have an accurate decision for the presence of the dip, we calculated the dispersion (i.e., standard deviation) of LF of the whole curve and the dispersion around the region of the dip which was suggested by Lee et al., 1997.

The results of these calculations are shown in Table 13. From this table, it is clear that the dispersion around the region where Wielen dip position was suggested is much smaller than the dispersion of the whole cluster, which assures the absence of any dip in the region of 7 mag. or 9 mag., except in Praesepe where the dispersion around the dip was slightly larger than the dispersion of the whole curve. The results obtained in the present work are in agreement 
TABLE 14: Luminosity functions for three open clusters and solar neighborhood stars due to Lee. et al., 1997.

\begin{tabular}{|c|c|c|c|c|c|c|c|c|c|}
\hline & -6 & -5 & -4 & -3 & -2 & -1 & 0 & 1 & 2 \\
\hline \multirow[t]{3}{*}{$M_{V}$} & 3 & 4 & 5 & 6 & 7 & 8 & 9 & 10 & 11 \\
\hline & 12 & 13 & 14 & 15 & 16 & 17 & 18 & 19 & 20 \\
\hline & & & & & & & & 4 & 19 \\
\hline \multirow[t]{3}{*}{ Hyades $\phi\left(M_{V}\right)$} & 30 & 32 & 33 & 49 & 48 & 45 & 34 & 49 & 86 \\
\hline & 18 & 162 & 96 & 79 & 66 & 7 & 23 & 10 & \\
\hline & & & & & & & & 3 & 20 \\
\hline \multirow[t]{3}{*}{ Praesepe $\phi\left(M_{V}\right)$} & 23 & 59 & 63 & 67 & 82 & 78 & 68 & 114 & 200 \\
\hline & 221 & 148 & 146 & 94 & 6 & & & & \\
\hline & & & & & & & 11 & 19 & 35 \\
\hline \multirow[t]{3}{*}{ Pleiades $\phi\left(M_{V}\right)$} & 41 & 58 & 73 & 77 & 62 & 69 & 95 & 137 & 182 \\
\hline & 198 & 182 & 64 & 10 & & & & & \\
\hline & 0.219 & 0.71 & 2.028 & 5.228 & 11.618 & 20.470 & 30.487 & 33.429 & 36.402 \\
\hline \multirow[t]{2}{*}{ Field star $\cdot \phi\left(M_{V}\right) \times 30$} & 48.431 & 66.546 & 74.838 & 69.202 & 58.901 & 74.152 & 81.306 & 109.678 & 195.039 \\
\hline & 263.100 & 138.077 & 97.751 & 53.718 & 35.491 & 30.912 & 24.554 & 9.335 & 2.885 \\
\hline
\end{tabular}

Solar neighborhood field stars.

TABLE 15: The dispersions in comparison with absolute value of the dip in each cluster.

\begin{tabular}{lccc}
\hline Cluster name & $\begin{array}{c}\text { Dispersion of } \\
\text { the LF curve of } \\
\text { the whole } \\
\text { cluster }\end{array}$ & $\begin{array}{c}\text { Dispersion at } \\
\text { the dip region }\end{array}$ & $\begin{array}{c}\text { Amplitude due } \\
\text { to dip region }\end{array}$ \\
\hline Hyades & 0.431 & 0.246 & 0.1587 \\
Praesepe & 0.525 & 0.214 & 0.10701 \\
Pleiades & 0.4 & 0.127 & 0.0464 \\
\hline \multicolumn{4}{c}{ TABLE 16: The Stock 2 IMF. } \\
\hline$M_{V} \quad \log (\mathcal{M} / \mathcal{M} \odot)$ & $\log \xi(\log \mathcal{M})$ \\
\hline-2.257 & 1.007 & -2.496 \\
-0.541 & 0.761 & -1.402 \\
1.176 & 0.528 & -0.459 \\
2.892 & 0.307 & 0.331 \\
4.609 & 0.098 & 0.971 \\
6.325 & -0.099 & 1.459 \\
8.041 & -0.284 & 1.795 \\
9.758 & -0.457 & 1.980 \\
11.474 & -0.617 & 1.895 \\
13.191 & -0.765 & \\
\hline
\end{tabular}

with those obtained by Miller and Scalo [8], where they concluded that there is no physical significance of this dip because its size is within the errors of the determination of the LF, and the dip is not found by all investigators.

The absence of any dip in the LF of the studied clusters in the present work makes us more interested to use Lee's original data [15] for calculating the general dispersion of the figure and the dispersion around the suggested magnitude intervals of the dip.

We redraw Lee's data of 1997 presented in Table 14 as shown in Figure 2 for the three open clusters, Hyades (closed
TABLE 17: The NGC 869 (h-Per) IMF.

\begin{tabular}{lcc}
\hline$M_{V}$ & $\log \left(\mathcal{M} / \mathcal{M}_{\odot}\right)$ & $\log \xi(\log \mathcal{M})$ \\
\hline-6.237 & 1.623 & -5.616 \\
-4.484 & 1.344 & -4.141 \\
-2.731 & 1.077 & -2.824 \\
-0.977 & 0.823 & -1.666 \\
0.776 & 0.581 & -0.665 \\
2.529 & 0.352 & 0.177 \\
4.282 & 0.136 & 0.861 \\
6.036 & -0.067 & 1.387 \\
7.789 & -0.258 & 1.755 \\
9.542 & -0.436 & 1.965 \\
11.296 & -0.601 & 2.017 \\
13.049 & -0.754 & 1.910 \\
14.802 & -0.893 & 1.646 \\
\hline
\end{tabular}

triangle), Praesepe (opened square), and Pleiades (opened circle) with solar neighborhood (closed circle), keeping Lee's scale in our drawings. Comparing Figure 2 with Lee's Figure 3 which is copied from the paper published by Lee et al. (1997). It was found that there is a mistake in his Figure 3 regarding to the Pleiades cluster. Into which the LF of the Pleiades cluster lies above the LF of the solar neighborhood stars and below the LF of the Hyades cluster while in Figure 2 the LF of the Pleiades cluster is above all the LF curves of the other open clusters in most parts, and it lies between Hyades and Praesepe clusters starting from $M_{V} \simeq 15 \mathrm{mag}$.

We calculate the dispersions of the LF curve of the whole cluster and the region around the suggested dip for these three open clusters given by Lee et al. in 1997. The results are shown in Table 15, from which it is clear that the dispersion around the region of the dip suggested by them is much smaller than the dispersion in the LF curve of the whole cluster, also its amplitude was approximately 
TABLe 18: The NGC 884 ( $\chi$-Per) IMF.

\begin{tabular}{lcc}
\hline$M_{V}$ & $\log \left(\mathcal{M} / \mathcal{M}_{\odot}\right)$ & $\log \xi(\log \mathcal{M})$ \\
\hline-3.558 & 1.201 & -3.426 \\
-1.999 & 0.969 & -2.322 \\
-0.440 & 0.747 & -1.342 \\
1.119 & 0.535 & -0.488 \\
2.679 & 0.334 & 0.241 \\
4.238 & 0.142 & 0.846 \\
5.797 & -0.040 & 1.325 \\
7.356 & -0.212 & 1.679 \\
8.915 & -0.374 & 1.908 \\
10.475 & -0.525 & 2.012 \\
12.034 & -0.667 & 1.991 \\
13.593 & -0.798 & 1.845 \\
15.152 & -0.920 & 1.574 \\
\hline
\end{tabular}

TABle 19: The NGC 2168 (M35) IMF.

\begin{tabular}{lcc}
\hline$M_{V}$ & $\log \left(\mathcal{M} / \mathcal{M}_{\odot}\right)$ & $\log \xi(\log \mathcal{M})$ \\
\hline 3.081 & 0.283 & 0.409 \\
4.641 & 0.094 & 0.981 \\
6.200 & -0.085 & 1.428 \\
7.759 & -0.255 & 1.750 \\
9.318 & -0.414 & 1.947 \\
10.877 & -0.563 & 2.019 \\
12.437 & -0.702 & 1.965 \\
\hline
\end{tabular}

Table 20: The NGC 2264 (S Mon).

\begin{tabular}{lcc}
\hline$M_{V}$ & $\log \left(\mathcal{M} / \mathcal{M}_{\odot}\right)$ & $\log \xi(\log \mathcal{M})$ \\
\hline-2.023 & 0.973 & -2.338 \\
0.699 & 0.592 & -0.706 \\
3.420 & 0.241 & 0.544 \\
6.141 & -0.079 & 1.414 \\
8.863 & -0.368 & 1.902 \\
11.584 & -0.627 & 2.010 \\
\hline
\end{tabular}

TABLE 21: The NGC 2516 IMF.

\begin{tabular}{lcc}
\hline$M_{V}$ & $\log \left(\mathcal{M} / \mathcal{M}_{\odot}\right)$ & $\log \xi(\log \mathcal{M})$ \\
\hline-1.583 & 0.909 & -2.048 \\
0.475 & 0.622 & -0.826 \\
2.532 & 0.352 & 0.178 \\
4.590 & 0.100 & 0.965 \\
6.648 & -0.135 & 1.533 \\
8.705 & -0.352 & 1.884 \\
10.763 & -0.552 & 2.018 \\
\hline
\end{tabular}

measured based on shape of the curve around the dip, it is clear that these amplitude values are within the dispersion around the dip region as shown in Table 15. This makes us on the side of the nonreality of the suggested dip. Especially, if we know that Lee et al. have included white dwarfs in
TABle 22: The NGC 2632 (Praesepe, M44) IMF.

\begin{tabular}{lcc}
\hline$M_{V}$ & $\log \left(\mathcal{M} / \mathcal{M}_{\odot}\right)$ & $\log \xi(\log \mathcal{M})$ \\
\hline 0.006 & 0.686 & -1.085 \\
1.648 & 0.466 & -0.227 \\
3.290 & 0.257 & 0.493 \\
4.933 & 0.059 & 1.075 \\
6.575 & -0.127 & 1.517 \\
8.217 & -0.302 & 1.821 \\
9.859 & -0.467 & 1.986 \\
11.501 & -0.620 & 2.012 \\
\hline
\end{tabular}

TABLE 23: The NGC 3532 IMF.

\begin{tabular}{lcc}
\hline$M_{V}$ & $\log \left(\mathcal{M} / \mathcal{M}_{\odot}\right)$ & $\log \xi(\log \mathcal{M})$ \\
\hline-1.438 & 0.888 & -1.955 \\
0.559 & 0.610 & -0.781 \\
2.555 & 0.349 & 0.188 \\
4.551 & 0.104 & 0.952 \\
6.548 & -0.124 & 1.511 \\
8.544 & -0.336 & 1.865 \\
10.540 & -0.531 & 2.014 \\
12.537 & -0.710 & 1.958 \\
\hline
\end{tabular}

TABLE 24: The Ruprecht 147 IMF.

\begin{tabular}{lcc}
\hline$M_{V}$ & $\log \left(\mathcal{M} / \mathcal{M}_{\odot}\right)$ & $\log \xi(\log \mathcal{M})$ \\
\hline 1.199 & 0.525 & -0.448 \\
3.265 & 0.260 & 0.483 \\
5.330 & 0.013 & 1.194 \\
7.396 & -0.216 & 1.686 \\
9.461 & -0.428 & 1.959 \\
11.527 & -0.622 & 2.012 \\
\hline
\end{tabular}

TABLE 25: The dispersions of the LF of the whole cluster compared with that of the dispersions around the dip region.

\begin{tabular}{lcc}
\hline Cluster name & $\begin{array}{c}\text { Dispersion of the } \\
\text { IMF curve of the } \\
\text { whole cluster }\end{array}$ & $\begin{array}{c}\text { Dispersion around } \\
\text { the dip region }\end{array}$ \\
\hline Stock 2 & 1.587 & 0.264 \\
NGC 869 (h-Per) & 2.568 & 0.286 \\
NGC 884 (c-Per) & 1.813 & 0.305 \\
NGC 2168 (M35) & 0.607 & 0.262 \\
NGC 2264 (S Mon) & 1.708 & 0.318 \\
NGC 2516 & 1.520 & 0.250 \\
NGC 2632 (Praesepe, & 1.136 & 0.238 \\
M44) & 1.458 & 0.258 \\
NGC 3532 & 0.968 & 0.387 \\
Ruprecht 147 &
\end{tabular}

their LF calculations, and LF as defined in the literature was not followed exactly by them since the volume was not considered in their calculations of LF. 


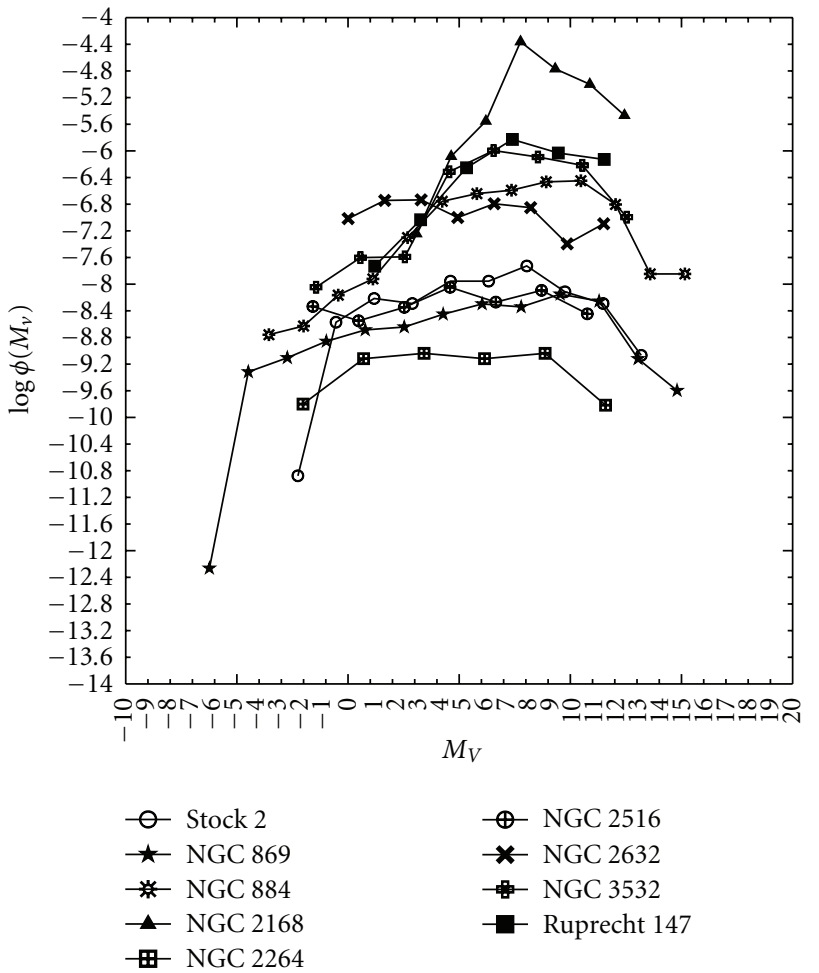

FIGURE 1: LFs of the present studied open clusters.

4.2. The Present Day Mass Function (PDMF) and IMF. The PDMF $\phi_{\mathrm{ms}}(\log \mathcal{M})$ is defined as the number of main sequence stars per unit logarithmic mass interval per square parsec in the solar neighborhood. The PDMF of main sequence field star is related to the LF of field stars $\phi\left(M_{V}\right)$ [8] by

$$
\phi_{\mathrm{ms}}(\log \mathcal{M})=\phi\left(M_{V}\right)\left|\frac{d M_{V}}{d \log \mathcal{M}}\right| 2 H\left(M_{V}\right) f_{\mathrm{ms}}\left(M_{V}\right),
$$

where $d M_{V} / d \log \mathcal{M}$ is the slope of the [ (absolute magnitude $M_{V}$, mass $\left.\mathcal{M}-\right)$ relation] and converts the LF to an MF. The term $2 \mathrm{H}\left(\mathrm{M}_{V}\right)$ is the result of integrating the LF perpendicular to the plane of the Galaxy, assuming an exponential distribution with scale height $H\left(M_{V}\right)$. The factor $f_{\mathrm{ms}}\left(M_{V}\right)$ gives the fraction of stars at a given magnitude which is on the MS. The values of the absolute magnitudes, masses, $d M_{V} / d \log \mathcal{M}$, scale heights, and the fraction $f_{\mathrm{ms}}$ adopted in the present investigations were given by Miller and Scalo [8].

The definition of the field star IMF in terms of all stars ever formed may seem confusing at first. However, in the case of a time-constant IMF (as assumed here), the IMF at any given time has the same shape as the IMF at any other time and, therefore, the same shape as the IMF of all stars ever formed. Note that the field star IMF as defined here and the IMF of stars in open clusters are similar quantities in that both give the mass distribution of stars at birth.

Stars with MS lifetimes greater than the age of the Galaxy will be found on the MS today regardless of when they were

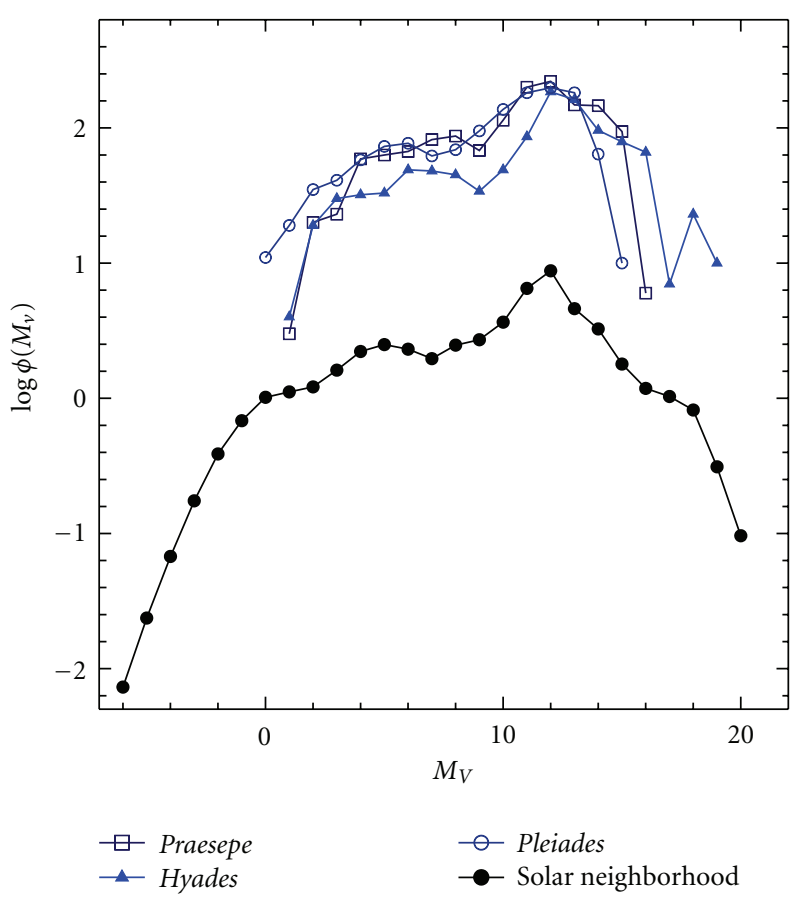

FIGURE 2: LFs for three open clusters and solar neighborhood stars after redrawing using Lee's data.

formed. For these stars (e.g., open clusters), the PDMF $\left(\phi_{\mathrm{ms}}(\log \mathcal{M})\right)$ and $\operatorname{IMF}(\xi(\log \mathcal{M}))$ are identical $[8,15]$ :

$$
\phi_{\mathrm{ms}}(\log \mathcal{M})=\xi(\log \mathcal{M}), \quad T_{\mathrm{ms}} \geq T_{0} .
$$

Now, we aimed to study the IMF of the above list of clusters (where the data were collected from ASCC-2.5 catalogue) to show if there is a Wielen dip at region $\log \mathcal{M} \approx$ $-0.25\left(\mathcal{M} \approx 0.56 \mathcal{M}_{\odot}\right)$ for Praesepe cluster and $\log \mathcal{M} \approx$ $-0.12\left(\mathcal{M} \approx 0.76 \mathcal{M}_{\odot}\right)$ for other open clusters [15]. Now, referring to (1) to calculate IMF for the above list of clusters, we get the data in Tables 16, 17, 18, 19, 20, 21, 22, 23, and 24 .

Again, we computed the dispersion of IMF and also the dispersion around the region of the dip (as a function of mass) which was suggested by Lee et al. [15].

The results of these calculations are shown in Table 25. Here, it is clear that the dispersion around the region of the Wielen dip which was suggested by Lee et al. [15] is much smaller than the dispersion of the whole cluster, which assure the absence of any dip in the region of $\log \mathcal{M} \approx-0.25(\mathcal{M} \approx$ $\left.0.56 \mathcal{M}_{\odot}\right)$ for Praesepe cluster and $\log \mathcal{M} \approx-0.12(\mathcal{M} \approx$ $\left.0.76 \mathcal{M}_{\odot}\right)$ for other open clusters. It is worthy to note that the dispersion of IMF of whole Praesepe cluster is much larger than the dispersion around the dip.

\section{Conclusion}

Finally, we concluded that the examination of IMF revealed the same results obtained with the LF, for example, there is no dip in the distribution of both LFs and IMFs. Also, Wielen [29] stated that most people explain the so-called Wielen dip in the LF of field stars as a consequence of a special property 


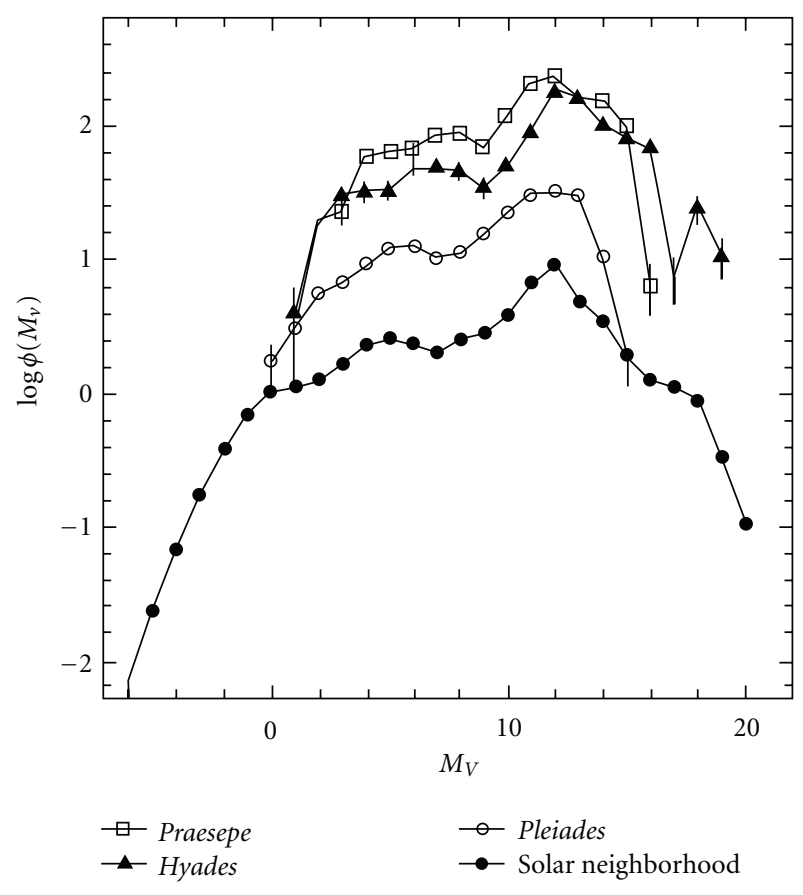

FIGURE 3: LFs for three open clusters and solar neighborhood stars, the original graph, copied from Lee et al. 1997.

of the relation between stellar mass and stellar luminosity in the region of the dip. If the IMF would be universal and valid for clusters too, then we should see the dip also in the LF of clusters. However, there are strong indications that the IMF in clusters differ from that of the field stars. In this case, the dip may not be strongly visible in the cluster LF.

\section{Acknowledgment}

The authors would like to thank Professor Dr. R. Wielen, for his communication and comments on this paper.

\section{References}

[1] R. Wielen, "The age distribution and total lifetimes of galactic clusters," Astron \& Astrophys, vol. 13, p. 309, 1971.

[2] R. E. Miller and J. M. Scalo, "On the birthplaces of stars," Physical Audio Signal Processing, vol. 90, p. 506, 1978.

[3] A. E. Piskunov, N. V. Kharchenko, S. Röser, E. Schilbach, and R. D. Scholz, "Revisiting the population of Galactic open clusters," Astronomy and Astrophysics, vol. 445, no. 2, pp. 545$565,2006$.

[4] A. E. Piskunov, N. V. Kharchenko, E. Schilbach, S. Röser, R. D. Scholz, and H. Zinnecker, "The initial luminosity and mass functions of the Galactic open clusters," Astronomy and Astrophysics, vol. 487, no. 2, pp. 557-566, 2008.

[5] R. Wielen, "The kinematics and ages of stars in Gliese's catalogue," in IAU Highlight of Astronomy, G. Contopoulos and G. Contopoulos, Eds., vol. 3, p. 395, Reidel, Dordrecht, The Netherlands, 1974.

[6] W. Gliese, "Katalog der Sterne näher als 20 Parsek für 1950.0," Mitt. Astron. Rechen-Institut, Heidelberg Serie A, no. 8, pp. 189, 1957.
[7] W. Gliese, "Catalogue of nearby stars. Edition," Veroffentl. Astron. Rechen-Institut, Heidelberg, no. 22, 1969.

[8] G. E. Miller and J. M. Scalo, "The initial mass function and stellar birthrate in the solar neighborhood," Astrophysical Journal Supplement Series, vol. 41, pp. 513-547, 1979.

[9] S. W. McCuskey, "The stellar luminosity function," Vistas in Astronomy, vol. 7, pp. 141-171, 1966.

[10] W. J. Luyten, "A new determination of the luminosity function," Monthly Notices of the Royal Astronomical Society, vol. 139, pp. 221-224, 1968.

[11] S.-W. Lee and M. Y. Chun, "Initial luminosity function and initial mass function for open clusters and associations," Journal of Korean Astronomical Society, vol. 21, p. 37, 1988.

[12] S. van den Bergh, "The luminosity function of star formation," The Astrophysical Journal, vol. 134, p. 553, 1961.

[13] R. B. Larson, "Turbulence and star formation in molecular clouds," Monthly Notices of the Royal Astronomical Society, vol. 194, p. 809, 1981.

[14] S.-W. Lee and H. Sung, "The luminosity function and initial mass function for the pleiades cluster," Journal of Korean Astronomical Society, vol. 28, p. 45, 1995.

[15] S.-W. Lee, H. Sung, and D.-H. Cho, "The luminosity function and mass function for the praesepe and hyades clusters," Journal of Korean Astronomical Society, vol. 30, p. 181, 1997.

[16] A. N. Belikov, S. Hirte, H. Meusinger, A. E. Piskunov, and E. Schilbach, "The fine structure of the Pleiades luminosity function and pre-main sequence evolution," Astronomy and Astrophysics, vol. 332, no. 2, pp. 575-585, 1998.

[17] A. E. Piskunov and A. N. Belikov, "Fine structure of the luminosity functions of young galactic clusters," Astronomy Letters, vol. 22, no. 4, p. 522, 1996.

[18] P. Kroupa, C. A. Tout, and G. Gilmore, "The low-luminosity stellar mass function," Monthly Notices of the Royal Astronomical Society, vol. 244, p. 76, 1990.

[19] F. D'Antona and I. Mazzitelli, "New pre-main-sequence tracks for $\mathrm{M} \leq 2.5 \mathrm{M}$ as tests of opacities and convection model," Astrophysical Journal, Supplement Series, vol. 90, no. 1, pp. 467-500, 1994.

[20] N. V. Kharchenko, "All-sky compiled catalogue of 2.5 million stars," Kinematics and Physics of Celestial Bodies, vol. 17, p. 409, 2001.

[21] N. V. Kharchenko, A. E. Piskunov, S. Röser, E. Schilbach, and R. D. Scholz, "Astrophysical supplements to the ASCC-2.5. II. Membership probabilities in 520 Galactic open cluster sky areas," Astronomische Nachrichten, vol. 325, no. 9, pp. 740-748, 2004.

[22] N. V. Kharchenko, A. E. Piskunov, S. Röser, E. Schilbach, and R. D. Scholz, "Astrophysical parameters of Galactic open clusters," Astronomy and Astrophysics, vol. 438, no. 3, pp. 11631173, 2005.

[23] N. V. Kharchenko, A. E. Piskunov, S. Röser, E. Schilbach, and R. D. Scholz, "109 new Galactic open clusters," Astronomy and Astrophysics, vol. 440, no. 1, pp. 403-408, 2005.

[24] P. J. van Rhijn, "The absorption of light in interstellar galactic space and the galactic density distribution," Publications of the Kapteyn Astronomical Laboratory Groningen, vol. 47, pp. 1-34, 1936.

[25] S.-W. Lee and H. S. Lee, "Luminosity functions and mass functions of eight open clusters," Journal of Korean Astronomical Society, vol. 17, p. 51, 1984.

[26] J. S. Kalirai, G. G. Fahlman, H. B. Richer, and P. Ventura, "The CFHT open star cluster survey. IV. Two rich, young open star clusters: NGC 2168 (M35) and NGC 2323 (M50)," Astronomical Journal, vol. 126, no. 3, pp. 1402-1414, 2003. 
[27] D. Barrado Y Navascués, J. R. Stauffer, J. Bouvier, and E. L. Martín, "From the top to the bottom of the main sequence: a complete mass function of the young open cluster M35," Astrophysical Journal, vol. 546, no. 2, pp. 1006-1018, 2001.

[28] J. Sanner and M. Geffert, "The IMF of open star clusters with Tycho-2," Astronomy and Astrophysics, vol. 370, no. 1, pp. 8799, 2001.

[29] R. Wielen, "Private communication," 2009. 

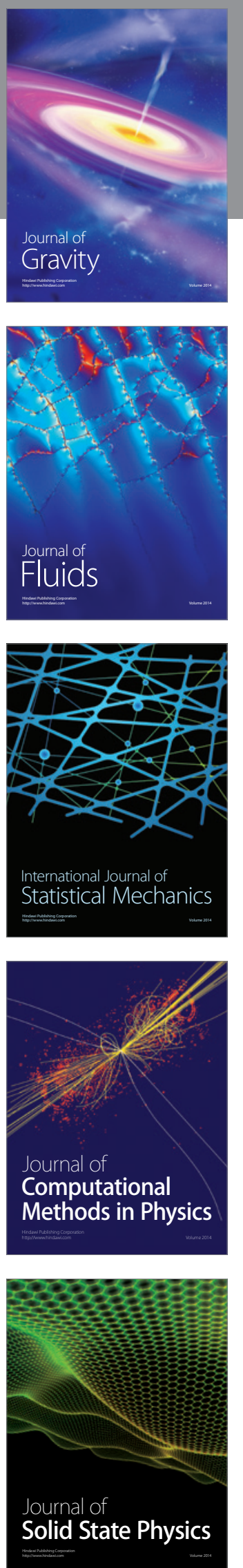

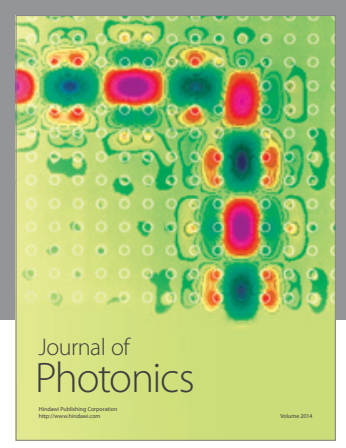

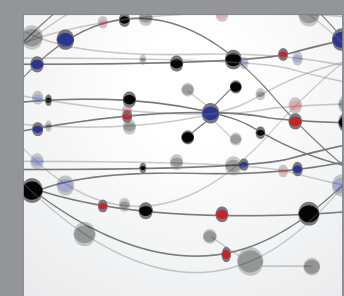

The Scientific World Journal
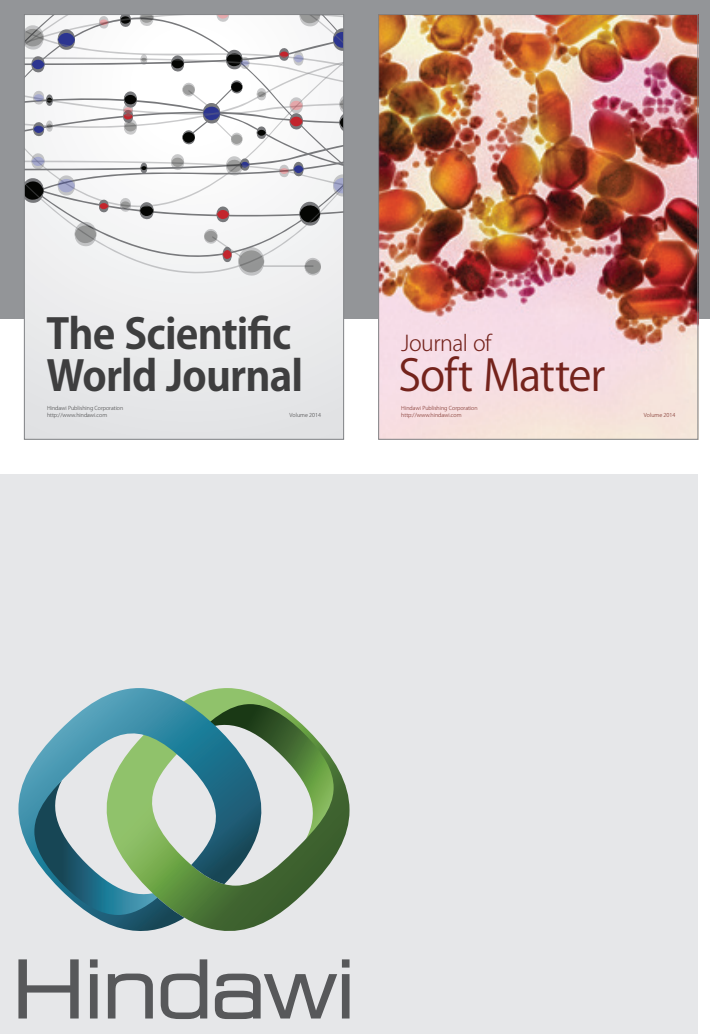

Submit your manuscripts at

http://www.hindawi.com
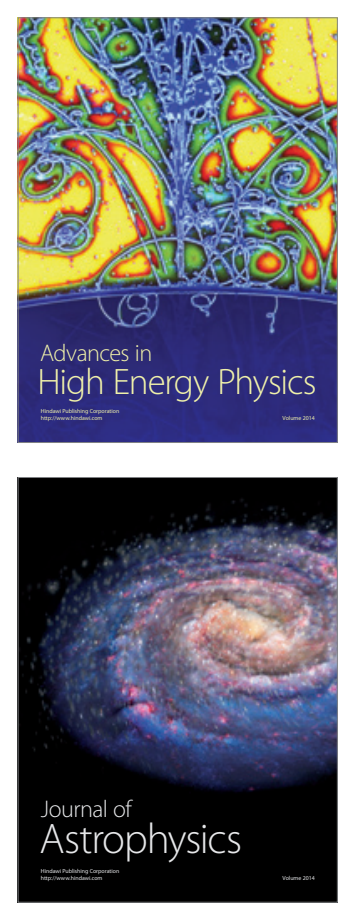
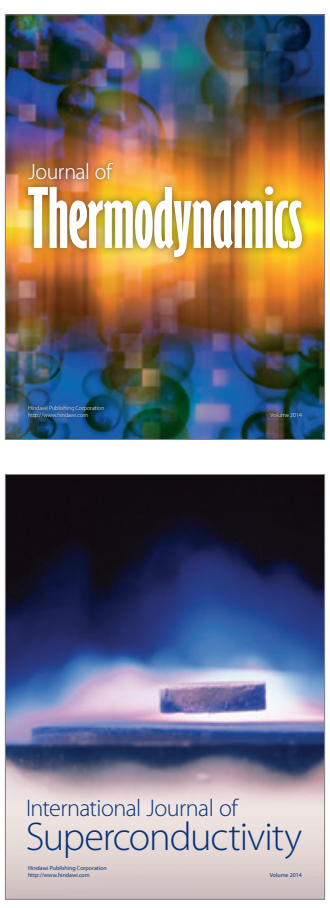
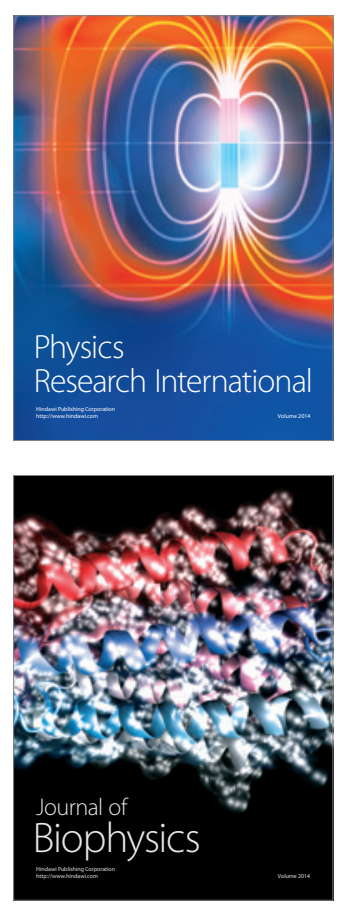
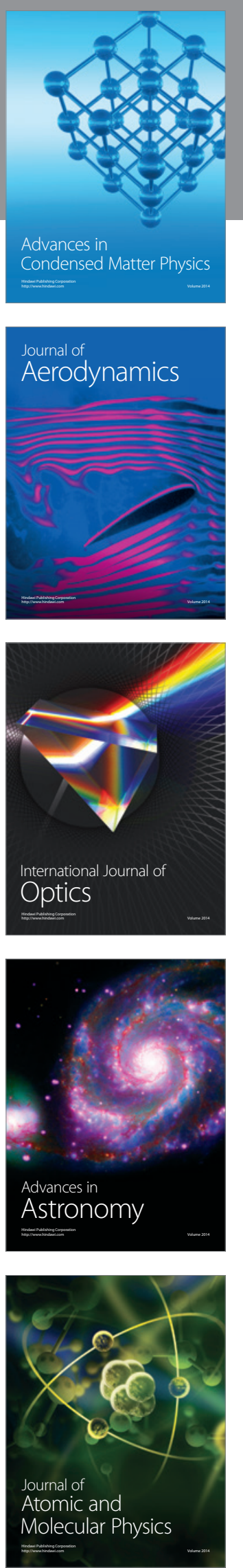